\title{
Warped extra dimension and inclined events at Pierre Auger Ob- servatory
}

\author{
Alexander Kisselev ${ }^{1, \star}$ and Liliya Shkalina ${ }^{2, \star \star}$ \\ ${ }^{1}$ Institute for High Energy Physics, NRC "Kurchatov Institute", 142281 Protvino, Russian Federation \\ ${ }^{2}$ Department of Physics, Moscow State University, 119991, Moscow, Russian Federation
}

\begin{abstract}
The generalized solution for the warp factor of the Randall-Sundrum metric is presented which is symmetric with respect to both branes and explicitly periodic in extra variable. Given that the curvature of the 5-dimensional space-time is small, the expected rate of neutrino-induced inclined events at the Surface Detector of the Pierre Auger Observatory is calculated. Both the "downward-going" (DG) and "Earth-skimming" (ES) neutrinos are considered. By comparing the expected event rate with the recent Auger data on searching for neutrino candidates, the lower bound on the fundamental gravity scale $M_{5}$ is obtained. The ratio of the number of the ES air showers to the number of the DG showers is estimated as a function of $M_{5}$.
\end{abstract}

\section{Introduction}

Ultra high energy (UHE) cosmic neutrinos play a key role in the determination of the composition of the ultra high energy cosmic rays (UHECRs) and their origin. UHE neutrinos are expected to be produced in astrophysical sources in the decays of charged pions created in the interactions of UHECRs with matter or radiation. They can be also produced via interaction of the UHECRs with the cosmic microwave background during propagation to the Earth (cosmogenic neutrinos). UHE cosmic neutrinos are not deviated by magnetic fields and could point back to their sources.

Recently, three neutrinos of energy 1-2 PeV, as well as tens of neutrinos above $10 \mathrm{TeV}$ were detected with the IceCube experiment [1]. The cosmic neutrinos with energies near $1 \mathrm{EeV}$ are detectable with the Surface Detector (SD) of the Pierre Auger Observatory (PAO) [2]. In order to isolate neutrinoinduced events at the SD of the PAO, it is necessary to look for deeply penetrating quasi-horizontal (inclined) air showers [3]-[5]. The PAO can efficiently search for two types of neutrino-induced inclined air showers (see fig. 1):

1. Downward-going (DG) neutrino-induced showers. They are initiated by neutrinos moving with large zenith angle $\theta$ which interact in the atmosphere close to the SD. At the PAO the search is restricted to showers with $\theta>60^{\circ}$ [6]. Note that the background from hadronic showers above $10^{17} \mathrm{eV}$ is $\mathrm{O}(1)$ in 20 years, and it is negligible above $10^{19} \mathrm{eV}$ [7].

^e-mail: alexandre.kisselev@ihep.ru

$\star \star$ e-mail: la.shkalina@physics.msu.ru 
2. Earth-skimming (ES) showers induced by upward tau neutrinos. They can interact in the Earth's crust producing tau leptons. The latter are efficiently produced at zenith angles $90^{\circ}<\theta<95^{\circ}$ [6]. The tau leptons escape the Earth and decay in the atmosphere close to the SD [8]-[9].

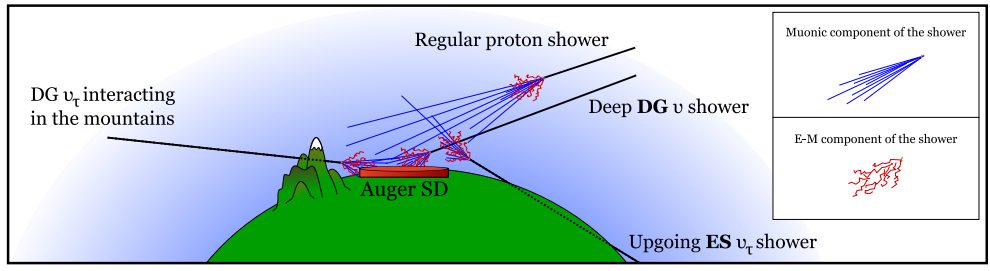

Figure 1. Different types of showers induced by DG and ES neutrinos (fig. 1 in [10]).

Recently, the Auger Collaboration reported on searches for DG neutrinos in the zenith angle bins $60^{\circ}-75^{\circ}$ and $75^{\circ}-90^{\circ}$, as well as for ES neutrinos. The data were collected by the SD of the PAO from 1 January 2004 until 20 June 2013 [6]. ${ }^{1}$ No neutrino candidates were found. Assuming the diffuse flux of UHE neutrinos to be $d N / d E_{v}=k E_{v}^{-2}$ in the energy range $1.0 \times 10^{17} \mathrm{eV}-2.5 \times 10^{19} \mathrm{eV}$, the stringent limit was obtained:

$$
k<6.4 \times 10^{-9} \mathrm{GeV} \mathrm{cm}^{2} \mathrm{~s}^{-1} \mathrm{sr}^{-1} .
$$

This Auger limit is a factor 3.64 below the Waxman-Bachall bound on neutrino production in optically thin astrophysical sources [11]:

$$
E_{v}^{2} \frac{d N}{d E_{v}}=2.33 \times 10^{-8} \mathrm{GeV} \mathrm{cm}^{2} \mathrm{~s}^{-1} \mathrm{sr}^{-1}
$$

In the Standard Model (SM) neutrino-nucleon cross sections are expected to be very small in comparison with hadronic cross sections even at UHEs [12]. That is why, the UHE cosmic neutrinos can be regarded as unique probes of new interactions. In the present paper a theory with an extra dimension (ED) is considered to be a "new physics" theory. We will see that effects coming from the ED can be significant or even dominant in the $v N$-scattering at UHEs.

\section{General solution for the Randall-Sundrum metric}

In ref. [13] the 5-dimensional space-time with non-factorizable geometry was suggested as an alternative to the models with flat extra dimensions. The Randall-Sundrum (RS) model [13] predicts an existence of heavy Kaluza-Klein (KK) excitations (massive gravitons). These massive KK resonances are intensively searched for at the LHC.

The RS scenario is described by the following background warped metric

$$
d s^{2}=e^{-2 \sigma(y)} \eta_{\mu \nu} d x^{\mu} d x^{v}-d y^{2},
$$

where $\eta_{\mu \nu}$ is the Minkowski tensor with the signature $(+,-,-,-)$, and $y$ is an extra coordinate. The periodicity condition $y=y+2 \pi r_{c}$ is imposed, and the points $\left(x_{\mu}, y\right)$ and $\left(x_{\mu},-y\right)$ are identified. Thus, one has a model of gravity in the $\mathrm{AdS}_{5}$ space-time compactified to the orbifold $S^{1} / Z_{2}$. The orbifold

\footnotetext{
${ }^{1}$ This search period is equivalent of 6.4 years of a complete Auger SD working continuously [6].
} 
has two fixed points, $y=0$ and $y=\pi r_{c}$. It is assumed that there are two branes located at these points. All the SM fields live on one of these branes.

The classical action of the RS scenario is given by [13]

$$
\begin{aligned}
S & =\int d^{4} x \int_{-\pi r_{c}}^{\pi r_{c}} d y \sqrt{G}\left(2 \bar{M}_{5}^{3} \mathcal{R}-\Lambda\right) \\
& +\int d^{4} x \sqrt{\left|g^{(1)}\right|}\left(\mathcal{L}_{1}-\Lambda_{1}\right)+\int d^{4} x \sqrt{\left|g^{(2)}\right|}\left(\mathcal{L}_{2}-\Lambda_{2}\right),
\end{aligned}
$$

where $G_{M N}(x, y)$ is the 5-dimensional metric, with $M, N=0,1,2,3,4, \mu=0,1,2,3$. The quantities

$$
g_{\mu \nu}^{(1)}(x)=G_{\mu \nu}(x, y=0), \quad g_{\mu \nu}^{(2)}(x)=G_{\mu \nu}\left(x, y=\pi r_{c}\right)
$$

are induced metrics on the branes, $\mathcal{L}_{1}$ and $\mathcal{L}_{2}$ are brane Lagrangians, $G=\operatorname{det}\left(G_{M N}\right), g^{(i)}=\operatorname{det}\left(g_{\mu \nu}^{(i)}\right)$. $\bar{M}_{5}$ is the reduced 5-dimensional Planck scale. The quantity $\Lambda$ is a 5-dimensional cosmological constant, while $\Lambda_{1,2}$ are brane tensions.

For the first time, the function $\sigma(y)$ was obtained in [13] (see also the expression at the bottom of fig. 2):

$$
\sigma_{0}(y)=\kappa|y|-C_{0},
$$

where $\kappa$ is a parameter with a dimension of mass, which defines the curvature of the 5-dimensional space-time. This solution is consistent with the orbifold symmetry $y \rightarrow-y$. However, it is attributed

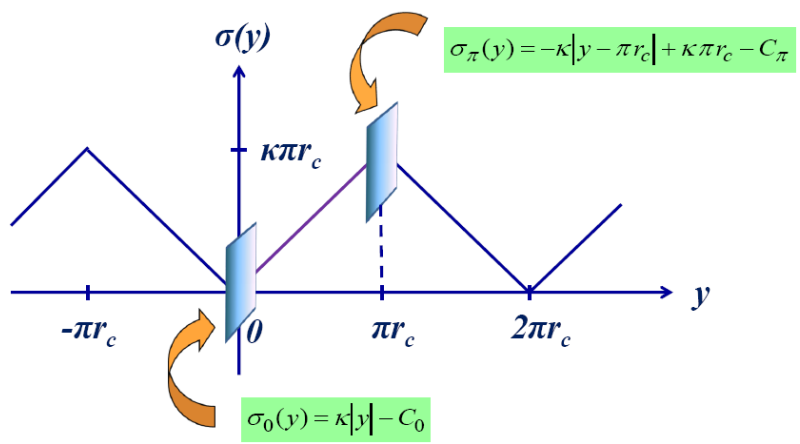

Figure 2. Two equivalent solutions of Einstein-Hilbert's equations for the function $\sigma(y)$ related to the different branes located at $y=0$ and $y=\pi r_{c}$.

to the brane $y=0$ (see fig. 2), and it is not symmetric with respect to the branes. ${ }^{2}$

Instead of (6), one can use another equivalent solution related to the brane $y=\pi r_{c}$ (see the expression at the top of fig. 2):

$$
\sigma_{\pi}(y)=-\kappa\left|y-\pi r_{c}\right|+|\kappa| \pi r_{c}-C_{\pi},
$$

In order to get an expression symmetric with respect to both branes, we take half the sum of expressions (6) and (7). As a result, we come to the solution:

$$
\sigma(y)=\frac{\kappa}{2}\left(|y|-\left|y-\pi r_{c}\right|\right)+\frac{|\kappa| \pi r_{c}}{2}-C,
$$

\footnotetext{
${ }^{2}$ Note that in contrast to (6), the RS1 solution [13] has no constant term.
} 
where $C=\left(C_{0}+C_{\pi}\right) / 2$. The constant terms in (8) are chosen in such a way that one has $\sigma(y)=\kappa y-C$ within the interval $0<y<\pi r_{c}$.

Note that neither original RS1 solution nor generalized RS-like solution (8) obeys periodicity in $y$ explicitly. One has to keep the periodicity condition in "mind". Fortunately, a new solution was recently proposed [14] which is both symmetric with respect to the branes and periodic function of $y$ :

$$
\sigma(y)=\frac{\kappa r_{c}}{2}\left[\left|\operatorname{Arccos}\left(\cos \frac{y}{r_{c}}\right)\right|-\left|\pi-\operatorname{Arccos}\left(\cos \frac{y}{r_{c}}\right)\right|\right]+\frac{\pi|\kappa| r_{c}}{2}-C,
$$

with the fine tuning relations:

$$
\Lambda=-24 \bar{M}_{5}^{3} \kappa^{2}, \quad \Lambda_{1}=-\Lambda_{2}=24 \bar{M}_{5}^{3} \kappa .
$$

Here $\operatorname{Arccos}(\mathrm{z})$ is a principal value of the multivalued inverse trigonometric function $\arccos (z)$. It is define by the inequality (see, for instance, [15]):

$$
0 \leqslant \operatorname{Arccos}(z) \leqslant \pi, \quad-1 \leqslant z \leqslant 1 .
$$

It means that [15]

$$
\operatorname{Arccos}(\cos x)= \begin{cases}x-2 n \pi, & 2 n \pi \leqslant x \leqslant(2 n+1) \pi, \\ -x+2(n+1) \pi, & (2 n+1) \pi \leqslant x \leqslant 2(n+1) \pi,\end{cases}
$$

where $n=0,1, \ldots$ It follows from (9), (12) that $\sigma(y)+C=\kappa y$ for $0<y<\pi r_{c}, \sigma(y)+C=\kappa\left(2 \pi r_{c}-y\right)$ for $\pi r_{c}<y<2 \pi r_{c}$. and so on (see fig. 2).

Our solution (9)

- is symmetric with respect to the branes: $\sigma(y)$ remains unchanged if $y \rightarrow \pi r_{c}-y, k \rightarrow-k ;^{3}$

- obeys the orbifold $Z_{2}$-symmetry: $\sigma(y)=\sigma(-y)$;

- reproduces the jumps of $\sigma^{\prime}(y)$ on both branes: $\sigma^{\prime \prime}(y)=2 \kappa\left[\delta(y)-\delta\left(y-\pi r_{c}\right)\right], 0 \leqslant y \leqslant \pi r_{c}$;

- is the periodic function of the extra coordinate: $\sigma\left(y+2 \pi r_{c} n\right)=\sigma(y), n= \pm 1, \pm 2, \ldots$ Note that the warped function $\sigma(y)$ depends on the constant $C$.

\section{RS-like scenario with the small curvature}

By taking different values of $C$ in eq. (9), we come to quite diverse physical scenarios [14], [16]. One of them $(C=0)$ is in fact the RS1 model [13]. Another scheme $\left(C=\kappa \pi r_{c}\right)$ describes a geometry with a small curvature of five-dimensional space-time (RSSC model) [17]-[19]. In the RSSC model the hierarchy relation looks like (we assume that $\kappa \pi r_{c} \gg 1$ )

$$
\bar{M}_{\mathrm{Pl}}^{2}=\frac{\bar{M}_{5}^{3}}{\kappa} e^{2 \kappa \pi r_{c}} .
$$

So, one can take, for instance, $\kappa \sim 1 \mathrm{GeV}, M_{5} \sim 1 \mathrm{TeV}$. Let us underline that the original RS scenario does not admit the parameters to lie in this region, since $\kappa \sim \bar{M}_{5} \sim M_{\mathrm{Pl}} \mathrm{TeV}$ in it [13].

The masses of the KK gravitons are proportional to the curvature parameter $\kappa$ :

$$
m_{n}=x_{n} \kappa,
$$

\footnotetext{
${ }^{3}$ Under the replacement $y \rightarrow \pi r_{c}-y$ the positions of the branes are interchanged $\left(y=0 \rightleftarrows y=\pi r_{c}\right)$, while under the replacement $k \rightarrow-k$ their tensions are interchanged $\left(\Lambda_{1} \rightleftarrows \Lambda_{2}\right)$.
} 
where $x_{n}$ are zeros of the Bessel function $J_{1}(x)$. The coupling of the massive gravitons to the SM fields is given by the constant:

$$
\Lambda_{\pi}=\bar{M}_{5}\left(\frac{\bar{M}_{5}}{\kappa}\right)^{1 / 2} .
$$

Thus, the RSSC scenario predicts a spectrum of the KK gravitons similar to that of the ADD model [20]-[21]. For the LHC phenomenology of the RSSC model, see [22], [23].

\section{Neutrino-nucleon amplitude in the RSSC model}

For the UHE cosmic neutrinos, the scattering takes place in the trans-Planckian kinematical region:

$$
\sqrt{s} \gtrsim \bar{M}_{5} \gg-t
$$

with $\sqrt{s}$ being the colliding energy and $t=-q_{\perp}^{2}$ four-dimensional momentum transfer. Remember that the inequality $\kappa \ll \bar{M}_{5}$ is also satisfied. In the eikonal approximation, which is valid in the kinematical region (16), the neutrino-proton amplitude is of the form:

$$
A_{\nu \mathrm{p}}(s, t)=4 \pi i s \int_{0}^{\infty} d b b J_{0}\left(b q_{\perp}\right)\{1-\exp [i \chi(s, b)]\} .
$$

In its turn, the eikonal is given by the Fourier-Bessel transformation of the eikonal:

$$
\chi(s, b)=\frac{1}{4 \pi s} \int_{0}^{\infty} d q_{\perp} q_{\perp} J_{0}\left(q_{\perp} b\right) A_{v \mathrm{p}}^{\mathrm{B}}(s, t) .
$$

The Born amplitude is given by the sum of gravi-Reggeons, i.e. reggeized gravitons in the $t$ channel. Because of a presence of the ED, the Regge trajectory of the graviton is splitting into an infinite sequence of trajectories enumerated by the KK number $n$ [24]:

$$
\alpha_{n}(t)=2+\alpha_{g}^{\prime} t-\alpha_{g}^{\prime} m_{n}^{2}, \quad n=0,1, \ldots
$$

In string theories, the slope of the gravi-Reggeons is universal, and $\alpha_{g}^{\prime}=M_{s}^{-2}$, where $M_{s}$ is the string scale. As a result, in the RSSC model the gravity Born amplitude for the neutrino scattering off $a$ point-like particle looks like [18], [25]:

$$
A_{\text {grav }}^{\mathrm{B}}(s, t)=\frac{\pi \alpha_{g}^{\prime} s^{2}}{2 \Lambda_{\pi}^{2}} \sum_{n \neq 0}\left[i-\cot \frac{\pi \alpha_{n}(t)}{2}\right]\left(\frac{s}{\bar{M}_{5}}\right)^{\alpha_{n}(t)-2} .
$$

The hadronic Born amplitude in (18) is defined by a convolution of the gravity amplitude (20) and skewed ( $t$-dependent) PDFs $f_{i}\left(x, \mu^{2} ; t\right)$ :

$$
A_{\nu \mathrm{p}}^{\mathrm{B}}(s, t)=\sum_{i=q, \bar{q}, g} \int_{0}^{1} d x A_{\text {grav }}^{\mathrm{B}}(x s, t) f_{i}\left(x, \mu^{2} ; t\right) .
$$

We take the $t$-dependent PDFs in a factorized form:

$$
f_{i}\left(x, \mu^{2} ; t\right)=f_{i}\left(x, \mu^{2}\right) D(t) .
$$


For the PDFs $f_{i}\left(x, \mu^{2}\right)$ we use the CT14 parametrization [26] and put $\mu^{2}=|t|$. As for a suppression factor $D(t)$, at hadron scales it was evaluated to be $D(t)=\exp \left(t r_{0}^{2}\right)$, with $r_{0}^{2}=0.62 \mathrm{GeV}^{-2}$ [27]. For larger $|t|$, we assume $D(t)$ to have a power-like behavior. As a result, we come to the parametrization:

$$
D(t)= \begin{cases}\exp \left(t r_{0}^{2}\right), & |t| \leqslant q_{0}^{2}, \\ \left(\mathrm{e}|t| / q_{0}^{2}\right)^{-q_{0}^{2} r_{0}^{2}}, & |t|>q_{0}^{2},\end{cases}
$$

where $q_{0}=m_{\rho}$ is the $\rho$-meson mass, and ln $\mathrm{e}=1$.

The total cross sections with the account of the contribution from the KK gravitons are shown in fig. 3. We adopted the $\mathrm{SM} v N$ cross sections from [12]. One can see, the total cross section noticeably exceeds the SM one at energies higher than $10^{18}$ or $10^{19} \mathrm{eV}$, depending on the reduced 5-dimensional Planck scale $\bar{M}_{5}$.

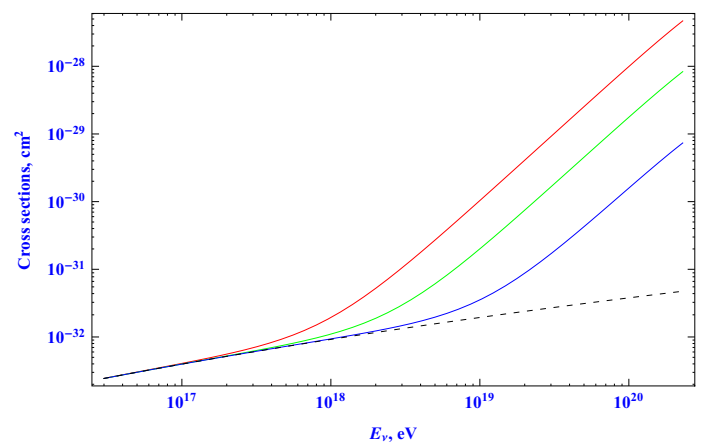

Figure 3. Solid lines (from above): the neutrino total cross sections for $\bar{M}_{5}=1.5,2.0$ and 3.0 TeV. Dashed line: neutrino CC total cross section.

\section{Expected number of neutrino events at the PAO}

In [7] the following functional dependence of the DG event rate on the new physics cross section $\sigma_{\mathrm{NP}}$ was proposed for a bin in neutrino energy $\left(10^{18.5} \mathrm{eV}<E_{v}<10^{19.5} \mathrm{eV}\right)$ :

$$
N_{\mathrm{BSM}}^{\mathrm{DG}}=N_{\mathrm{SM}}^{\mathrm{DG}} \frac{\sigma_{\mathrm{CC}}+\sigma_{\mathrm{NP}}}{\sigma_{\mathrm{CC}}},
$$

where $N_{\mathrm{SM}}^{\mathrm{DG}}$ is the number of DG events in the absence of new physics, $\sigma_{\mathrm{CC}}$ is a charged current $(\mathrm{CC})$ total cross section. $N_{\mathrm{BSM}}^{\mathrm{DG}}$ is the number of DG events with the account of interactions beyond the SM. We propose a more general formula:

$$
\mathcal{E}_{\mathrm{BSM}}^{\mathrm{DG}}\left(E_{v}\right)=\mathcal{E}_{\mathrm{SM}}^{\mathrm{DG}}\left(E_{v}\right) \frac{\sigma_{\mathrm{SM}}^{\mathrm{eff}}\left(E_{v}\right)+\sigma_{\mathrm{NP}}\left(E_{v}\right)}{\sigma_{\mathrm{SM}}^{\mathrm{eff}}\left(E_{v}\right)},
$$

where $\mathcal{E}_{\mathrm{BSM}}^{\mathrm{DG}}\left(\mathcal{E}_{\mathrm{SM}}^{\mathrm{DG}}\right)$ is the exposure of the $\mathrm{SD}$ of the $\mathrm{PAO}$ with (without) account of the new interaction. In addition, instead of $\sigma_{\mathrm{CC}}$, an effective SM cross section $\sigma_{\mathrm{SM}}^{\mathrm{eff}}$ is introduce in (25):

$$
\sigma_{\mathrm{SM}}^{\mathrm{eff}}=\sigma_{\mathrm{CC}} \sum_{i=e, \mu, \tau} m_{\mathrm{CC}}^{i}+3 \sigma_{\mathrm{NC}} m_{\mathrm{NC}}+\sigma_{\mathrm{CC}} m_{\text {mount }} .
$$


Here $m_{\mathrm{CC}}^{i}$ and $m_{\mathrm{NC}}$ are relative mass apertures for charged current and neutral current (NC) interactions of the DG neutrinos at the PAO. The mass aperture $m_{\text {mount }}$ corresponds to the CC interaction of a $\tau$ neutrino within the mountains around the PAO (see fig. 1). The relative mass apertures as functions of the neutrino energy where calculated using data from Table I of ref. [10]. ${ }^{4}$ Note that $\sum_{i=e, \mu, \tau} m_{\mathrm{CC}}^{i}+3 m_{\mathrm{NC}}+m_{\text {mount }}=1$.

In contrast to the DG neutrino exposure, the exposure of the ES neutrinos decreases with the rise of the neutrino total cross section: ${ }^{5}$

$$
\mathcal{E}_{\mathrm{BSM}}^{\mathrm{ES}}\left(E_{v}\right)=\mathcal{E}_{\mathrm{SM}}^{\mathrm{ES}}\left(E_{v}\right) \frac{\sigma_{\mathrm{CC}}^{2}\left(E_{v}\right)}{\left[\sigma_{\mathrm{CC}}\left(E_{v}\right)+\sigma_{\mathrm{NP}}\left(E_{v}\right)\right]^{2}} .
$$

The formulas (25) and (27) allowed us to calculate expected exposures of the SD of the PAO for the period 1 January 2004 - 20 June 2013. The Auger data on exposures for the SM neutrino interactions in the region from $\log \left(E_{v} / \mathrm{eV}\right)=17$ to 20.5 in steps of 0.5 were used (see also fig. 3 from [6]). The results of our calculations are presented in fig. 4.
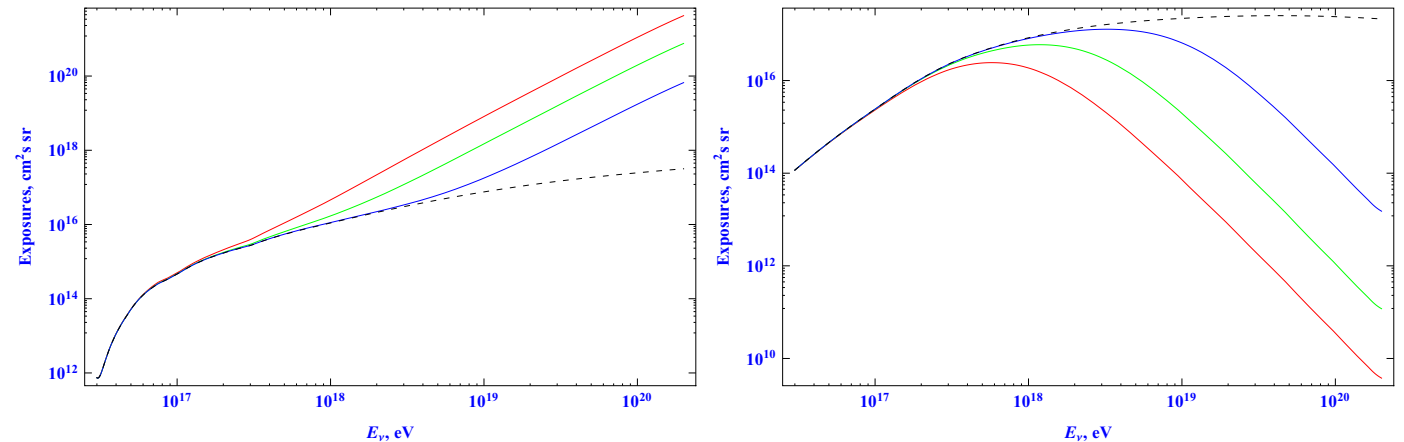

Figure 4. Left panel: expected Auger exposures for the DG neutrinos with zenith angle $75^{\circ}<\theta<90^{\circ}$. Red, green and blue line corresponds to $\bar{M}_{5}=1.5,2.0$ and $3.0 \mathrm{TeV}$, respectively. Dashed line: SM exposure taken from [6]. Right panel: the same but for the ES neutrinos.

The number of neutrino events is given by the formula:

$$
N_{\mathrm{ev}}=\int \frac{d N_{v}}{d E_{v}}\left(E_{v}\right) \mathcal{E}\left(E_{v}\right) d E_{v} .
$$

We assume differential neutrino flux $d N / d E_{v} \sim E_{v}^{-2}$, as well as a ratio $v_{e}: v_{\mu}: v_{\tau}=1: 1: 1$. In fig. 5 we compare the number of neutrino events predicted in the RSSC model with the expected number of neutrino events in the absence of a new physics. Both the DG events with zenith angles $60^{\circ}<\theta<90^{\circ}$ and ES events was taken into account in the full energy region of the sensitivity of the $\mathrm{SD}\left(1.0 \times 10^{17}-2.5 \times 10^{19} \mathrm{eV}\right)$. As was already mentioned above, no neutrino events were seen at the $\mathrm{SD}$ of the PAO. Thus, from fig. 5 we obtain the lower bound on 5-dimensional reduced Planck scale:

$$
\bar{M}_{5}>2.75 \mathrm{TeV} \text {. }
$$

Correspondingly, for the 5-dimensional Planck scale we find $M_{5}>2.75 \times(2 \pi)^{1 / 3}=5.07 \mathrm{TeV}$.

We have also calculated an expected ratio of the ES neutrino showers to the DG neutrino showers with the zenith angles $75^{\circ}<\theta<90^{\circ}$ (see fig. 6). We predict $N_{\mathrm{ES}} / N_{\mathrm{DG}}$ to be 2.8 (5.7) for $\bar{M}_{5}=2.8$

\footnotetext{
${ }^{4}$ In particular, $\sum_{i=e, \mu, \tau} m_{\mathrm{CC}}^{i}$ is equal to $0.69(0.65)$ at $10^{18}\left(10^{19}\right) \mathrm{eV}$.

${ }^{5}$ Is is a generalization of the formula for the rate for ES showers in the range $10^{18.5} \mathrm{eV}<E_{v}<10^{19.5} \mathrm{eV}$ [7].
} 


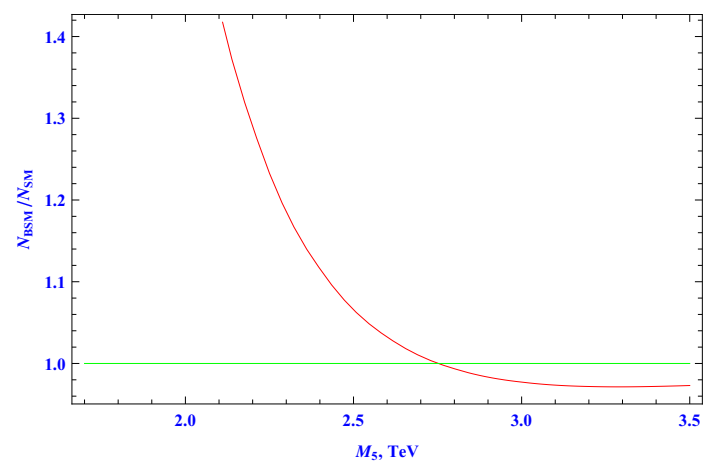

Figure 5. The ratio of the neutrino events with and without effects from EDs as a function of the gravity scale $\bar{M}_{5}$, expected at the SD of the PAO. The event numbers correspond to the energy range $1.0 \times 10^{17}-2.5 \times 10^{19} \mathrm{eV}$.

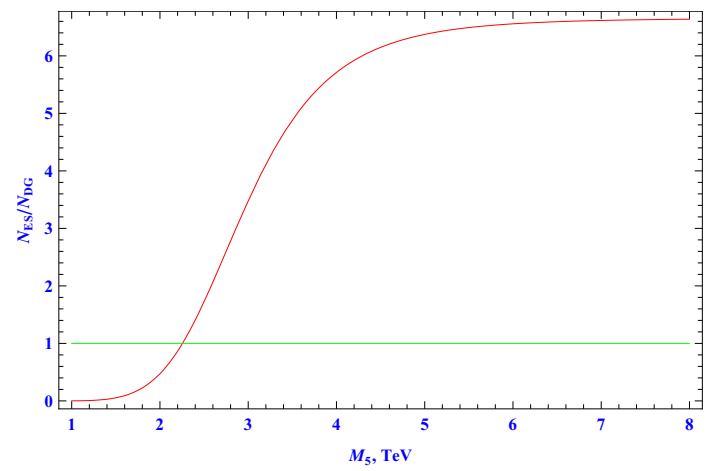

Figure 6. The expected ratio of the ES neutrinos to the DG neutrinos (with zenith angle $75^{\circ}<\theta<90^{\circ}$ ) at the SD of the PAO as a function of the gravity scale $M_{5}$.

(4.0) TeV. For the SM interactions, this ratio was estimated by the Auger Collaboration to be equal to $6.0[6]$.

\section{Conclusions}

In the present paper we have studied the neutrino-induced inclined (quasi-horizontal) events at the Surface Detector of the Pierre Auger Observatory in the Randall-Sundrum scenario with the extra dimension and warped metric. We have presented the general solution for the metric (9) which is symmetric with respect to both branes and explicitly periodic in extra variable. In the framework of the RS-like model with the small curvature of the 5-dimensional space-time, the exposures of the SD of the PAO for the downward going and Earth-skimming neutrinos are estimated (fig. 4). The lower bound on the fundamental gravity scale $\bar{M}_{5}$ is obtained (29). The ratio of the number of the ES air showers to the number of the DG showers, $N_{\mathrm{ES}} / N_{\mathrm{DG}}$, is calculated as a function of $\bar{M}_{5}$ (fig. 6). 


\section{Acknowledgements}

The authors are indebted to J. Alvarez-Muñiz for sending numerical data on the Auger exposures shown in fig. 3 from ref. [6].

\section{References}

[1] M.G. Aartsen et al. (IceCube Collab.), Phys. Rev. Lett. 113, 101101 (2014)

[2] J. Abraham et al. (Auger Collab.), Nucl. Instrum. Meth. A 523, 50 (2004)

[3] V.S. Berezinsky and G.T. Zatsepin, Phys. Lett. B 28, 423 (1969); V.S. Berezinsky and A.Yu. Smirnov, Astrophys. Space Sience 32, 461 (1975)

[4] K.S. Capelle, J.W. Cronin, G. Parente and E. Zas, Astropart. Phys. 8, 321 (1998)

[5] E. Zas, New J. Phys., 7, 130 (2005)

[6] A. Aab et al. (Auger Collab.), Phys. Rev. D 91, 092008 (2015)

[7] L. Anchordoqui, H. Goldberg, D. Góra et al., Phys. Rev. D 82, 043001 (2010)

[8] X. Bertou et al., Astropart. Phys. 17, 183 (2002)

[9] J.L. Feng, P. Fisher, F. Wilczek and T.M. Yu, Phys. Rev. Lett. 88, 161102 (2002)

[10] P. Abreu et al. (Auger Collab.), Phys. Rev. D 84, 122005 (2011)

[11] E. Waxman and J.N. Bachall, Phys. Rev. D 64, 023002 (2001)

[12] A. Cooper-Sarkar and S. Sarkar, JHEP 0801, 075 (2008)

[13] L. Randall and R. Sundrum, Phys. Rev. Lett. 83, 3370 (1999)

[14] A.V. Kisselev, Proc. of the Third Annual Large Hadron Collider Physics Conference (LHCP2015), August 31-September 2, 2016, St. Petersburg, Russia

[15] I.S. Gradshteyn and I.M. Ryzhik, Table of Integrals, Series, and Products, Eighth Edition (Eds. D. Zwillinger and V. Moll, Academic Press, US, 2014) 55-56.

[16] A.V. Kisselev, Generalization of the Randall-Sundrum solution, arXiv:1512.01091, Nucl. Phys. B 909 (2016), to appear.

[17] G. F. Giudice, T. Plehn and A. Strumia, Nucl. Phys. B 706, 455 (2005)

[18] A.V. Kisselev and V.A. Petrov, Phys. Rev. D 71, 124032 (2005)

[19] A.V. Kisselev, Phys. Rev. D 73, 024007 (2006)

[20] N. Arkani-Hamed, S. Dimopoulos and G. Dvali, Phys. Lett. B 429, 263 (1998); Phys. Rev. D 59, 086004 (1999)

[21] I. Antoniadis, N. Arkani-Hamed, S. Dimopoulos and G. Dvali, Phys. Lett. B 436, 257 (1998)

[22] A.V. Kisselev, JHEP 0809, 039 (2008)

[23] A.V. Kisselev, JHEP 1304, 025 (2013)

[24] A.V. Kisselev and V.A. Petrov, Eur. Phys. J. C 36, 103 (2004); ibid 37, 241 (2004)

[25] A.V. Kisselev, Open Astron. J. 2, 12 (2009)

[26] S. Dulat et al., Phys. Rev. D 93, 033006 (2016)

[27] V.A. Petrov and A.V. Prokudin, Eur. Phys. J. C, 23, 135 (2002) 